\title{
Development and Application of Portable Reference Radiation Fields with Multi-Point Single-Energy Photon
}

\author{
Lin Quan1, Hongchun Shan', Zhe Dai', Jianhui Lo², Haisheng Zhou'², Yan Zhang1 \\ ${ }^{1}$ Beijing Institute of Tracking and Telecommunications Technology, Beijing, China \\ ${ }^{2}$ Northwest Institute of Nuclear Technology, Xi'an, China \\ Email: jlxsd@163.com
}

Received 29 April 2015; accepted 13 October 2015; published 20 October 2015

\begin{abstract}
Based on the needs of detection and calibration with low-energy X-ray, we used the relationship characteristics of $\mathrm{X}$-ray fluorescence, absorption and limit potential target excitation, developed the X-ray device with adjustable intensity (single photon to $10^{7} / \mathrm{s}$ ), optional energy points (4 keV $20 \mathrm{keV}$ ), highly portable ( $\leq 1 \mathrm{~kg}$ ), by the matching design of fluorescence energy conversion target and modulation means, coupled transport simulation of electron-photon in target, meanwhile, we solved the low-energy X-ray radiation field diagnose problems with HPGe detector, which calibrated with combined technique, including relatively wide energy efficiency simulation and single energy point of absolute efficiency calibration. In single-photon calibration field of soft X-ray pulsar navigation detect, the portable reference single energy radiation fields was applied effectively and got good result, the reference radiation fields provided perfect experiment means for the scientific study of pulsar navigation detecting, sun X-ray monitoring, etc.
\end{abstract}

\section{Keywords}

Low-Energy X-Ray, Monokinetic, Characteristics Fluorescence, Absorption Edge, Filter

\section{Introduction}

It is the urgent need to acquire a portable low energy single-photon radiation with accurate parameter, including energy and fluence, to support the quantum efficiency calibration for detector and weak signal discriminated study in these fields, such as pulsar navigation detection, faint target characteristics distinguish, blackbody radiation diagnostics and so on [1]-[6]. Usually there are three ways to obtain radiation field, First, by X-ray fluorescence, narrow spectrum, broad spectrum of X-ray machine in the calibration laboratory, in order to service instrumentation dose or dose rate calibration of radiation protection field, its energy monochromaticity is poor [7] [8]. Secondly, by synchrotron radiation, it has advantages in good monochromaticity and adjustable intensity, but its huge volume (unable motion), high running cost and limited resource, which bring huge inconvenient in experiment. Third, by isotope radiation's characteristic X-rays from nuclear decay, for instance of Am241, Fe56, etc., it is difficult to avoid interfere $\mathrm{X}$-ray of high energy. In our work explore the technique of material absorp-

How to cite this paper: Quan, L., Shan, H.C., Dai, Z., Lo, J.H., Zhou, H.S. and Zhang, Y. (2015) Development and Application of Portable Reference Radiation Fields with Multi-Point Single-Energy Photon. Journal of Applied Mathematics and Physics, 3, 1231-1238. http://dx.doi.org/10.4236/jamp.2015.310151 
tion edge modulation method and measuring technique, to develop producing technology of low energy single photon with miniature X-ray machines, and application in detector calibrations [9] [10].

\section{The Device Development}

\subsection{The Structure and Principle}

The single energy X-ray photon device developed is composition of X-ray photon machine (A), shield bracket (B), fluorescence conversion target (C), intensity adjustment sheet (D), collimator (E), filter sheet (F) and monitoring detector, the schematic layout shown in Figure 1, the three process of X-ray energy modulation schematic is shown in Figure 2.

\subsection{Theoretical Simulation}

In order to modulate X-ray energy according to three stages as shown in Figure 2, the device structure designed was shown in Figure 1. We used simulation means of empirical formula and Monte Carlo to obtain the required modulation parameters, such as X-ray machine set voltage, absorbent material species, thickness and so on.

(1) Primary radiation. The primary radiation is produced from ionizing $(-d E / d x)_{\text {ion }}$ and radiation $(-d E / d x)_{\text {rad }}$ in the anode target with high speed electronics incident, which are composition with characteristic fluorescence (separate energy) and bremsstrahlung (continuous energy) [11] [12]. These spectrum and intensity of two kinds of X-ray are decided by the incident electron energy, angle and target material, the corresponding expression of cross sections see Formulas (1) and (2).

$$
\begin{gathered}
\left(-\frac{d E}{d x}\right)_{i o n}=\frac{2 \pi e^{4} N Z}{m_{0} v^{2}}\left[\operatorname{Ln} \frac{m_{0} v^{2}}{I}-1.2329\right] \\
\left(-\frac{d E}{d x}\right)_{\text {rad }} \propto \frac{z^{4} Z^{2}}{m^{2}} N E
\end{gathered}
$$

In the formula, $v$ is electronic speed, $\mathrm{m} / \mathrm{s}$. $E$ is electron energy, MeV. $x$ is an electronic walking distance, $\mathrm{m} . m$ is the electron mass, kg. $z$ is electronic charge number, C. $Z$ is the atomic number of the target substance. $N$ is atomic density of the material in per-unit volume, $\mathrm{m}^{-3}$.

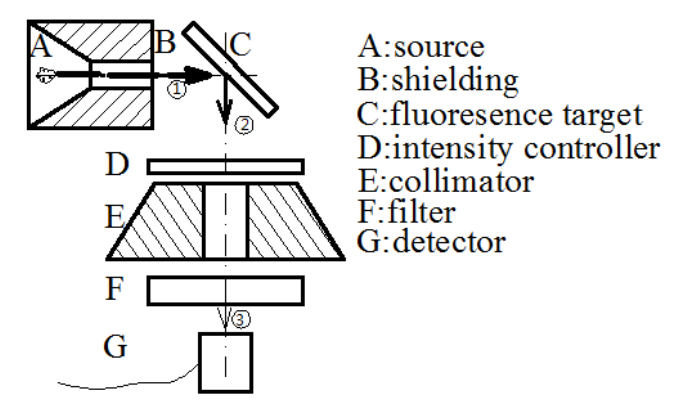

Figure 1. The structure layout sketch of fluorescence conversion device.

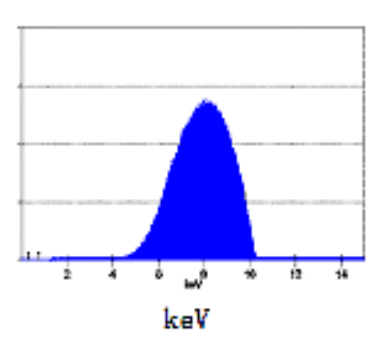

(a)

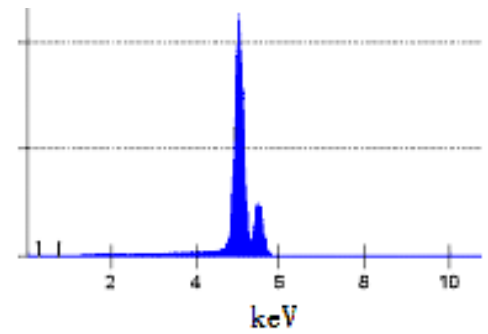

(b)

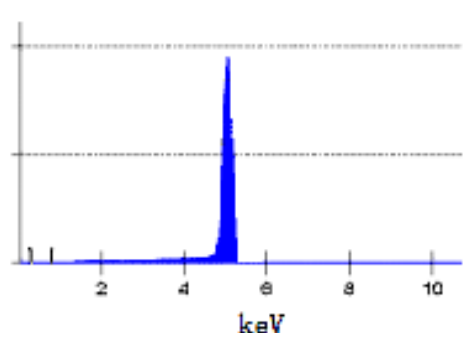

(c)

Figure 2. The stages schematic of Spectrum changes. (a) Primary radiation; (b) Fluorescence radiation; (c) Filtration radiation. 
Based on the above empirical formulas, in low-energy region, the bremsstrahlung and fluorescence yield would increase with the increase of the incident photon energy, but the share of high energy in bremsstrahlung would increase, which also would affect monochromatic radiation of target photon. It is necessary to seek a balance between the fluorescence yield and scattering interference. In our work, under the condition of different setting voltages with electrons accelerated in $20 \mu \mathrm{m}$ tungsten target and $250 \mu \mathrm{m}$ beryllium target, using the simulation means of particle transport code MCNP [13], we simulated the primary X-ray spectrum, the simulation curve see in Figure 2, the results can provide data base for subsequent acceleration voltage setting.

(2) Fluorescence radiation. The primary X-rays incident in the target, which deposited energy in the target, and produced fluorescence photons, scattered photons and heat mainly through the photoelectric effect and Compton effect. According to the atomic energy level distribution and incident photon energy characteristics, in the low-energy region, the main fluorescence excited is $k$ layer, and its cross section $\sigma_{p h, k}$ is determined by the atomic number and the incident photon, the relationship shown in Equation (3). Because of the soft X-ray energy is lower $\left(E_{x}<<m_{0} c^{2}\right)$, in this case, the basic component of Compton scattering composition come from Thomson scatter, whose scattering cross section can be simplified to Equation (4).

$$
\begin{gathered}
\sigma_{p h, k}=Z^{5}(1 / h v)^{(2 / 7)} \\
\sigma_{c}=8 / 3 \pi Z r_{0}^{2}
\end{gathered}
$$

In the formula, $r_{0}$ is classical radius of the electron, about $2.8 \times 10^{-13} \mathrm{~cm}$.

From the Formula (3), the photoelectric cross section increase obviously with atomic number $\mathrm{Z}$ increases of target material, and reduce with incident photon energy increases. The Formula (4) shown the cross sections is independent with the photon energy, but is inversely proportional to the atomic number, it can clearly be seen that the energy of incident photon is to decisive parameters of fluorescence energy. In simulation, the X-ray fluorescence spectrum at different set voltage was shown in Figure 4, under the conditions of the initial radiation spectrum shown in Figure 3, structure and layout as described in Figure 1, the thick copper of fluorescent target C is $200 \mu \mathrm{m}$, the copper thickness of intensity control sheet $\mathrm{D}$ is $50 \mu \mathrm{m}$.

(3) Radiation filtered. As shown in Figure 1, when the X-ray of characteristic fluorescence distribution in (2) of Figure 4 penetrate fluorescence filter $\mathrm{F}$, the photon attenuation will obey exponentially rule as shown in Equation 5 . Due to the fluorescent to be modulated is composed by $\mathrm{k}_{\alpha}$ and $\mathrm{k}_{\beta}$ (L fluorescence yield is very low, can be ignored), in the energy modulation, we select these characteristic target material as filter, which of excitation potential is slightly larger than $\mathrm{k}_{\alpha}$ or $\mathrm{k}_{\beta}$, meanwhile, the energy absorbing edge is near the target $\mathrm{k}_{\beta}$ in the middle of $\mathrm{k}_{\alpha}$ and $\mathrm{k}_{\beta}$ energy. In order to make full use of material absorb coefficient mutation's characteristic to strengthen photon absorption in region near $\mathrm{k}_{\beta}$ energy, and to adjust the fluorescence spectra of transmission, using iterative simulation method to determine the thickness $d$. The following describe of the $\mathrm{Cu}$ target as an example,

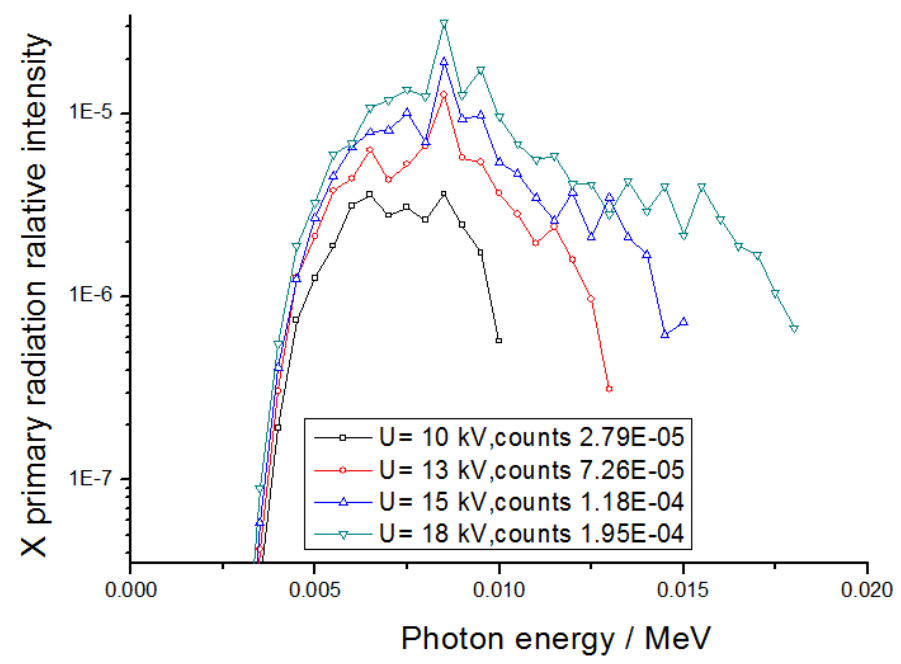

Figure 3. The primary radiation spectrum simulated. 


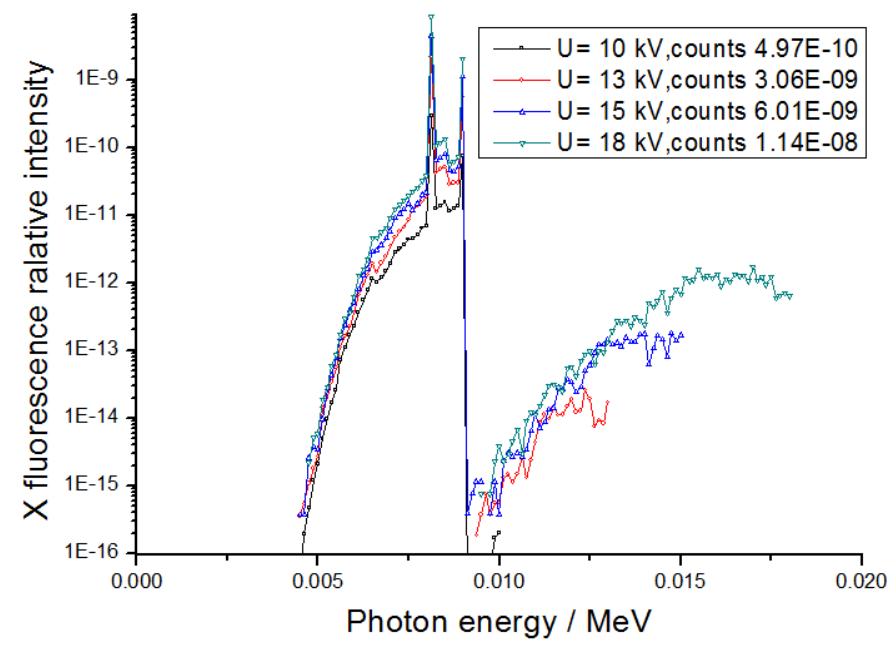

Figure 4. The fluorescence radiation spectrum simulated.

$\mathrm{K}_{\alpha 1}$ energy is $8.047 \mathrm{keV}, \mathrm{K}_{\alpha 2}$ energy is $8.027 \mathrm{keV}, \mathrm{K}_{\beta}$ energy is $8.940 \mathrm{keV}$, L fluorescent energy are $0.928 \mathrm{keV}$ and $0.948 \mathrm{keV}$. Selecting $\mathrm{Ni}$ as the filter is more appropriate, the excitation potential of the corresponding energy is slightly higher than $8.940 \mathrm{keV}$, suitable for $\mathrm{K}_{\beta}$ energy copper absorption fluorescence. In our simulations by MCNP program, when Ni target is setting 50 um, the spectrum with absorption before and after obtained can see as Figure 5.

$$
I=I_{0} e^{-\mu d}
$$

Figure 5 shows that the $\mathrm{K}_{\beta}$ energy of $\mathrm{Cu}$ fluorescence $(8.940 \mathrm{keV}$ ) can absorb effectively with fluorescence modulated, and the interference components can been suppressed down nearly one orders of magnitude, the average energy of primary spectrum can modulated from $8.30 \mathrm{keV}$ to $8.13 \mathrm{keV}$. Under these conditions, the total energy peak region is between $8 \mathrm{keV}-8.15 \mathrm{keV}$, which is obtained from spectrum modulated by the HPGe detector, the probability of peak count is up to $98 \%$ in total spectrum, and the energy monochromatic better than 98.2\%, this can be very suitable for low-energy detector energy calibration. According to this principle and simulation methods within $4 \mathrm{keV}-15 \mathrm{keV}$ energy range, in our work selection several monoenergetic photon of the $4.58 \mathrm{keV}, 4.95 \mathrm{keV}, 8.04 \mathrm{keV}, 16.58 \mathrm{keV}$ to design, the detailed parameter can see below summarizes.

\subsection{Structure Realization}

According to the configuration shown in Figure 1, using MOXTEK company's TUB00082 type X-ray tube as primary fluorescence excitation source, that can run at voltages between 4 - $50 \mathrm{kV}$ current 0 - $200 \mu \mathrm{A}$, focal spot is $300 \mu \mathrm{A}$, anode target is $\mathrm{W}$, thick beryllium window is $250 \mu \mathrm{m}$, the power supply is DC $24 \mathrm{~V}$, the maximum power is $4 \mathrm{~W}$. In order to convenience of experimental application and strengthening radiation protection, we set the $1 \mathrm{~cm}$ Fe shielding to control the transmittance rate of $50 \mathrm{keV}$ X-ray within $10^{-4}$ level out of shielding, through MCNP simulation, and develope the fluorescent device with 45 degrees conversion target. The device is composed of a scattering target, collimator and absorbing sheet, and the power supply and control systems are encapsulated in the control box.

\section{Radiation Field Diagnose}

Obtaining accurate parameters is the basis for device applications, in our study, selection of Canberra HPGe GL0213R type as a diagnostic device, using a combination means of theoretical and experimental, to implement system calibration and measurement experiments.

\subsection{Measure System Calibration}

The calibration of energy and efficiency is data foundation to ensure detection data reliable. For low-energy detector, the serious nonlinearity of detection efficiency and lack of multi-point energy calibration source, which 


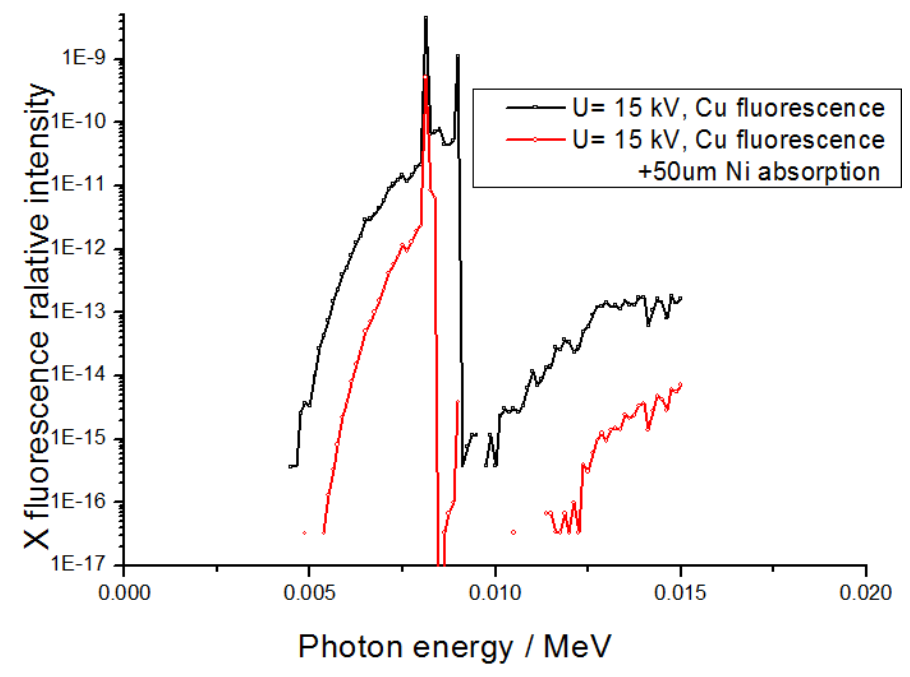

Figure 5. The filtered fluorescence spectrum simulated.

brought great difficulties for the absolute fluence of the radiation field measurements. In our work, according to the X-ray energy range, using the Monte Carlo method to get the relative detection efficiency curve, combined with absolute calibration method of Am-241 standard radioactive source, we gain the absolute detection efficiency curve with the relative efficiency's absolute calibration, the efficiency curve of the detector in setting energy region was shown in Figure 6.

\subsection{Measurement Results and Analysis}

Using the measure system, we complete the X-ray radiation field measure under various operating conditions, the parameters of fluorescence spectrum and fluence rate were summarized in Table 1, the spectrum and fluence in the several typical condition of X source running measured are seen in Figure 7.

Shown in Table 1, using fluorescence converting target and energy modulators, we can provide favorable radiation field with different energy and fluence rate in $4-20 \mathrm{keV}$, to meet the different sensitivity calibration of single photon detector. Figure 7 shows the various conditions to produce monochromatic radiation field is good, $5.01 \mathrm{keV}$ single photon obtained as target combinations of V-23 (vanadium) and Ti-22 (titanium), the energy resolving rate is better than $4 \% .8 .10 \mathrm{keV}$ single photon obtained as target combinations of $\mathrm{Ni}-28$ and $\mathrm{Cu}-29$, the Energy half-width can reach $0.4 \mathrm{keV}$. To more than $10 \mathrm{keV}$ photon, due to the fluorescence effect of X-ray machine's anode and shielding material, some interference photon maybe influence spectrum, such as combination with $\mathrm{Zr}-40$ and $\mathrm{Nb}-41$, the main energy of emission photon is $16.58 \mathrm{keV}$, but also exist other energy interfere photon, that should be paid attention in application.

\section{Trial Application}

According to the calibration task of single photon detector developed for the pulsar navigation radiation profile measurement [14], we build single photon measurement system with CR105PMT photomultiplier tube and TELEDYNE LECROY wave runner $610 \mathrm{Zi}$ oscilloscope. In order to deduct the oscilloscope baseline fluctuation interference, we used single pulse current integral method to realize the single photon signal record, and setted integral signal subtraction method with or without pulse signals. The experiment setting is as follows, the detector's high voltage is $-900 \mathrm{~V}$, the oscilloscope stall is $50 \mathrm{Mv} / \mathrm{div}$, trigger threshold is $1 \mathrm{mV}$, the test system structure is shown in Figure 8(a). Adjusting the radiation field parameters, control X-ray fluence in the $10^{7} / \mathrm{s}$ or less, on several conditions of single photon incident at $17.5 \mathrm{keV}, 8.43 \mathrm{keV}$ and $5.04 \mathrm{keV}$, the statistics of the detector response obtained was shown in Figure 8(b).

The calibration results of Figure 8 show that when there is no X-ray incidence, the oscilloscope recorded signal is composed with dark events, including cosmic rays, thermal noise, etc., the charge quantity forming with dark event is relatively large (the peak shown in a vertical line 1 , the relative charge quantity is $6.94 \times 10^{-10}$ 


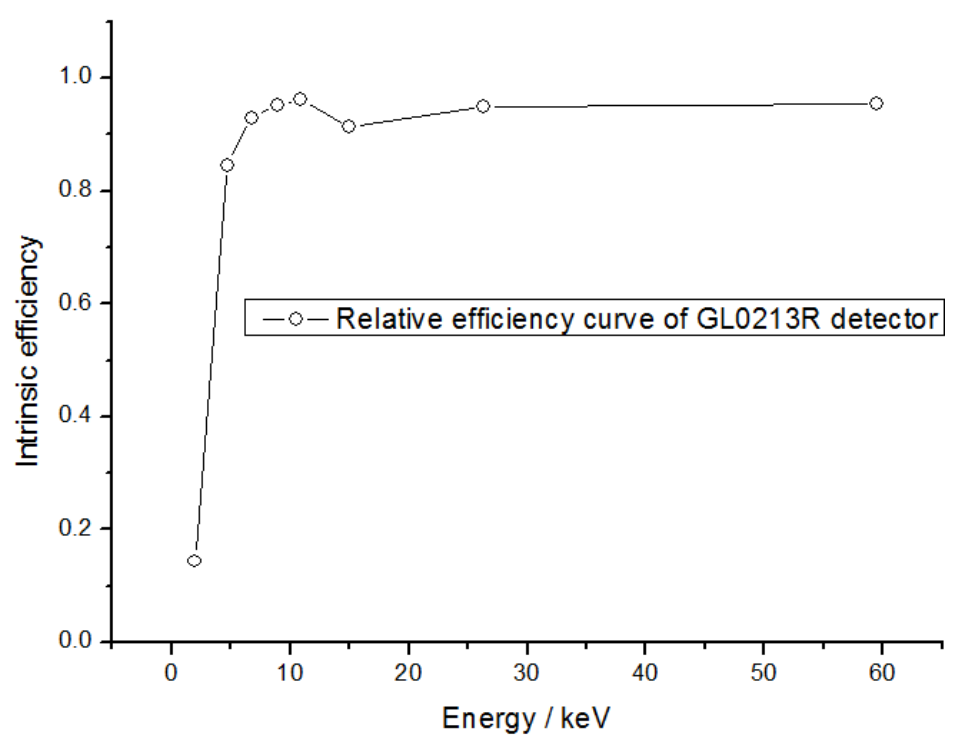

Figure 6. GL0213R type HPGe detector intrinsic efficiency curve.

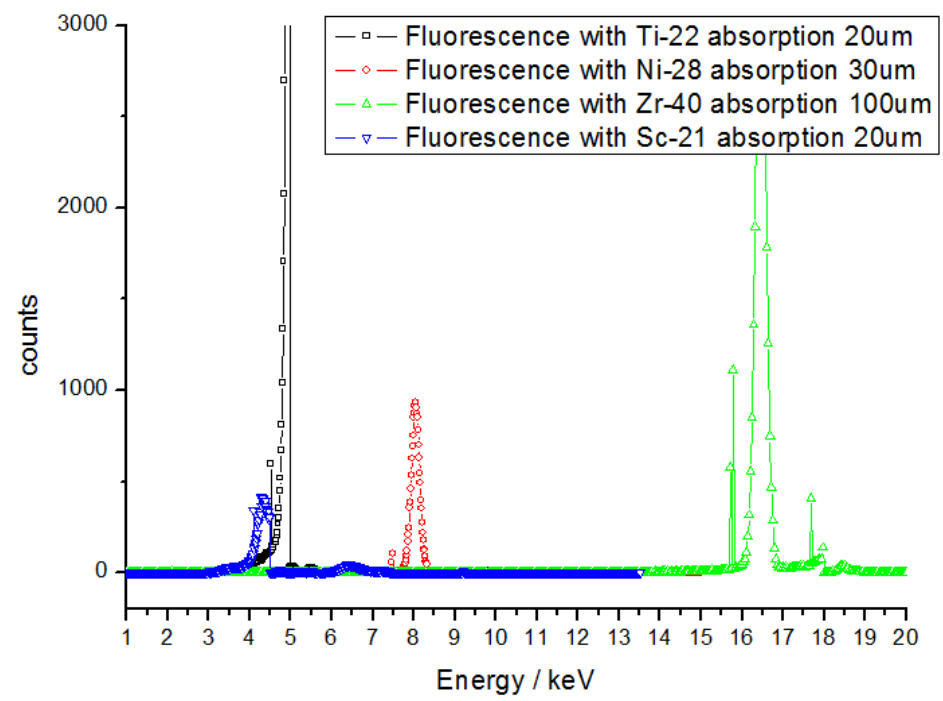

Figure 7. The single photon energy distribution established.

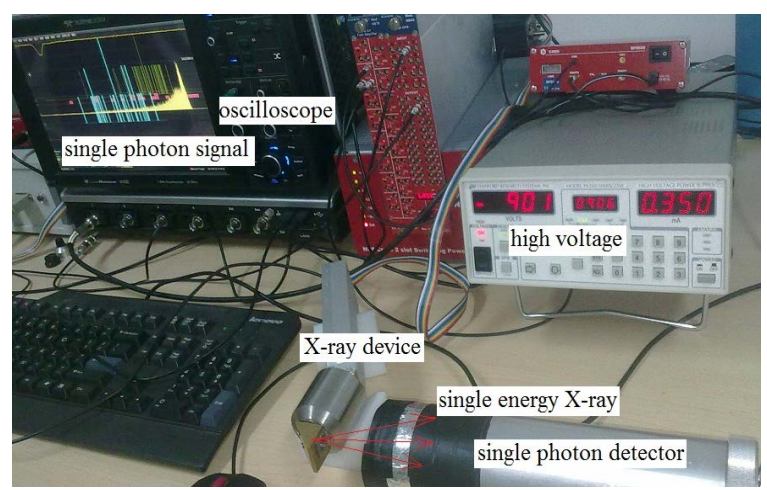

(a)

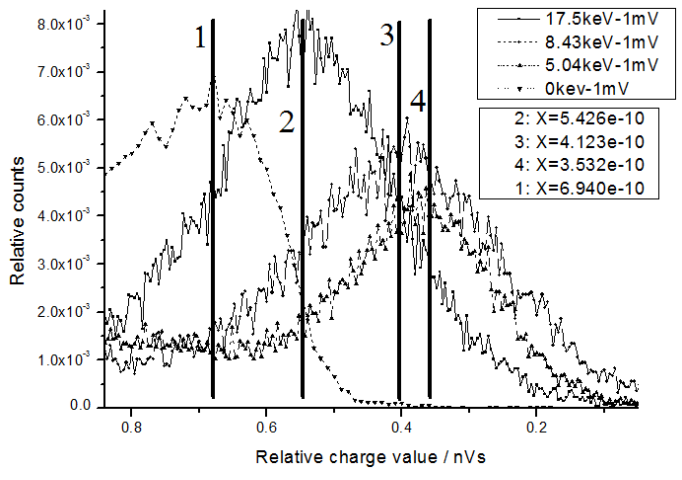

(b)

Figure 8. Different composite scintillator energy response of single photon energy calibration. 
Table 1. The summary of X-ray fluorescence condition and parameter.

\begin{tabular}{|c|c|c|c|c|c|c|}
\hline$E_{\text {average }}$ & $E_{\text {fluorescence }}$ (counts) & Running condition & Fluorescence target & Filter & $E_{\text {monokinetic }}$ & Fluence \\
\hline $4.58 \mathrm{keV}$ & $\begin{array}{l}\mathrm{K}_{\alpha}: 4.51 \mathrm{keV}(5914) \\
\mathrm{K}_{\beta}: 4.93 \mathrm{keV}(1251)\end{array}$ & $\begin{array}{l}10.9 \mathrm{kV} \\
9.6 \mu \mathrm{A}\end{array}$ & $\begin{array}{c}\mathrm{Ti}-22 \\
1 \times 20 \mu \mathrm{m}\end{array}$ & $\begin{array}{c}\text { Sc- } 21 \\
1 \times 20 \mu \mathrm{m}\end{array}$ & 4.52 & $1.49 \times 10^{3}$ \\
\hline $5.04 \mathrm{keV}$ & $\begin{array}{c}\mathrm{K}_{\alpha}: 4.95 \mathrm{keV}(18511) \\
\mathrm{K}_{\beta}: 5.43 \mathrm{keV}(4008)\end{array}$ & $\begin{array}{l}9.9 \mathrm{kV} \\
9.6 \mu \mathrm{A}\end{array}$ & $\begin{array}{c}\mathrm{V}-23 \\
1 \times 20 \mu \mathrm{m}\end{array}$ & $\begin{array}{c}\mathrm{Ti}-22 \\
1 \times 20 \mu \mathrm{m}\end{array}$ & 5.01 & $5.88 \times 10^{4}$ \\
\hline $8.43 \mathrm{keV}$ & $\begin{array}{c}\mathrm{K}_{\alpha}: 8.04 \mathrm{keV}(3352) \\
\mathrm{K}_{\beta}: 8.940 \mathrm{keV}(2534)\end{array}$ & $\begin{array}{l}14.9 \mathrm{kV} \\
99.6 \mu \mathrm{A}\end{array}$ & $\begin{array}{c}\mathrm{Cu}-29 \\
3 \times 100 \mu \mathrm{m}\end{array}$ & $\begin{array}{c}\mathrm{Ni}-28 \\
1 \times 30 \mu \mathrm{m}\end{array}$ & 8.10 & $1.67 \times 10^{4}$ \\
\hline $17.54 \mathrm{keV}$ & $\begin{array}{l}\mathrm{K}_{\alpha}: 16.58 \mathrm{keV}(11780) \\
\mathrm{K}_{\beta}: 18.62 \mathrm{keV}(10456)\end{array}$ & $\begin{array}{l}39.9 \mathrm{kV} \\
9.6 \mu \mathrm{A}\end{array}$ & $\begin{array}{c}\mathrm{Nb}-41 \\
3 \times 80 \mu \mathrm{m}\end{array}$ & $\begin{array}{c}\mathrm{Zr}-40 \\
1 \times 100 \mu \mathrm{m}\end{array}$ & 16.60 & $3.65 \times 10^{4}$ \\
\hline
\end{tabular}

$\mathrm{nVs}$ ). When X-ray radiating, the dark counts share of detector by oscilloscope records is very down, the response waveform show good characteristics of Gauss distribution. When the single photon incident energy at $17.5 \mathrm{keV}$, the signal peak shown in vertical line 2, the relative charge quantity is $5.426 \times 10^{-10} \mathrm{nVs}$. When the single photon incident energy at $8.43 \mathrm{keV}$, the signal peak shown in the vertical line 3 , the relative charge quantity is $4.123 \times 10^{-10} \mathrm{nVs}$. When the single photon incident energy at $5.04 \mathrm{keV}$, the signal peak shown in the vertical line 4 , the relative charge quantity is $3.532 \times 10^{-10} \mathrm{nVs}$.

The results of application show that, single photon detection signal increase with the incident energy increase, but the signal range change exhibit nonlinear with energy increases. There are many factor could produce above result, such as the detector dark current, fluorescence conversion efficiency of different energy X-ray, energy scatter on detector surface with reflective film, these issues requires careful study in subsequent detector calibration application. It is obvious that the multi-single energy photon obtained are significance for detector calibration, and need to develope highly portable single photon device for the application, the reference radiation field can provide ideal conditions for the calibration of the detector quantum efficiency calibration and so on.

\section{Summary}

This study based on the micro X-ray fluorescence and filtering technology, through fluorescence modulation matching design of conversion target and absorbing target, coupled transport simulation of electron and photon in the target material, device structure and shielding design, etc., and combined with the theoretical simulation and experimental measurement techniques, the desired optimum excitation voltage of each single fluorescent produced and the thickness of absorption film were explored, and GL0213R type HPGe detector efficiency calibration problem was solved, and the emitted photon energy and fluence rate were diagnosis, at the same time, each characteristics of signal energy X-ray spectrum obtained were evaluated. The diagnostic results shows that the portable single-photon source of energy was good selectivity between $4 \mathrm{keV}-20 \mathrm{keV}$, such as $4.51 \mathrm{keV}$, $5.01 \mathrm{keV}, 8.10 \mathrm{keV}, 16.60 \mathrm{keV}$ etc. The half width energy of $5.01 \mathrm{keV}$ photon is about $0.2 \mathrm{keV}$ with adjustable strength in $10-10^{4} / \mathrm{s}$, it is suitable for different sensitivity detector calibration. At the same time, the minisize $\mathrm{X}$-ray device has a good portability and radiation safety, the volume is not more than $10 \mathrm{~cm} \times 15 \mathrm{~cm} \times 10 \mathrm{~cm}$. It is the ideal tool for single photon detector response calibrated, has broad application prospects in many lowenergy photons and detection, calibration and signal screening and other research.

\section{References}

[1] Tibolla, O., Kaufmann, S. and Kosack, K. (2014) XMM-Newton and Chandra X-Ray Follow-Up Observations of the VHE Gamma-Ray Source HESS J1507-622. HESS J1507-622 X-Ray Follow-Ups, 1-9.

[2] Mike, G.B., Werner, B., Tobias, P., et al. (2011) Autonomous Spacecraft Navigation Based on Pulsar Timing Information. Presented at the 2nd International Conference on Space Technology, Athens, 15-17 September. arXiv: 1111.1138v1.

[3] Chen, B.M., Zhao, B.S., Hu, H.J., et al. (2011) X-Ray Photon-Counting Detector Based on a Micro-Channel PLATE for Pulsar Navigation. Chinese Optics Letters, 9, Article ID: 060401.

[4] Zheng, L. and Wei, C. (2014) Development of Novel On-Chip, Customer-Design Spiral Biasing Adaptor on for Si Drift Detectors and Detector Arrays for X-Ray and Nuclear Physics Experiments. Nuclear Instruments and Methods in Physics Research A, 765, 17-22. http://dx.doi.org/10.1016/j.nima.2014.06.068 
[5] Sheikh, S.I., Pines, D.J., et al. (2004) The Use of X-Ray Pulsar for Spacecraft Navigation. Proceedings of the 14th AAS/AIAA Space Flight Mechanics Conference, AAS 04-109, Maui, Feb, 105-119.

[6] Shpilman, Z., Ehrlich, Y., Maman, S., et al. (2014) Single-Shot Calibration of Soft X-Ray Mirrors Using a Sinusoidal Transmission Gratinga. Review of Scientific Instruments, 85, 11E809. http://dx.doi.org/10.1063/1.4890404

[7] ISO 4037-1 (1996) X and Gamma Reference Radiation for Calibrating Dose Meters and Doserate Meters and for Determining Their Response as a Function of Photon Energy-Part 1: Radiation Characteristics and Production Methods. First Edition, 1996.12.15. Switzerland, 17-21.

[8] Maderitsch, A., Smolek, S., Wobrauschek, P., et al. (2014) Feasibility Study of Total Reflection X-Ray Fluorescence Analysis Using a Liquid Metal Jet X-Ray Tube. Spectrochimica Acta Part B, 99, 67-69. http://dx.doi.org/10.1016/j.sab.2014.06.003

[9] Abbey, B., Wlutehead, L.W., Quiney, H.M., et al. (2011) Lensless Imaging Using Broadband X-Ray Sources. Nature Photon, 5, 420-424. http://dx.doi.org/10.1038/nphoton.2011.125

[10] Rachevski, A., Zampa, G., Zampa, N., et al. (2014) Large-Area Linear Silicon Drift Detector Design for X-Ray Experiments. Journal of Instrumentation, 9.

[11] Maderitsch, A., Smolek, S., Wobrausche, P., et al. (2014) Feasibility Study of Total Reflection X-Ray Fluorescence Analysis Using a Liquid Metal Jet X-Ray Tube. Spectrochimica Acta Part B, 99, 67-69. http://dx.doi.org/10.1016/j.sab.2014.06.003

[12] Zoukel, A. and Khouchaf, L. (2014) The Secondary X-Ray Fluorescence and Absorption near the Interface of MultiMaterial: Case of EDS Microanalysis. Micron, 67, 81-89. http://dx.doi.org/10.1016/j.micron.2014.06.009

[13] Briesmeister, J.F. (1997) MCNP-A General Monte Carlo N-Partical Transpont Code. Ver. 4B, LA12625-M. Los Alamos Scientific Laboratory, New Mexico, 1.1-4.58.

[14] Quan, L. and Ouyang, X.-P. (2014) Developing Review of X-Ray Detection Technology for Pulsar Navigation. Modern Applied Physics, 5, 98-103. (In Chinese) 